\title{
Hidden curriculum - was ist denn das?
}

\section{Werner Bauer}

Dr. med., Präsident des Schweizerischen Instituts für ärztliche Weiter- und Fortbildung SIWF

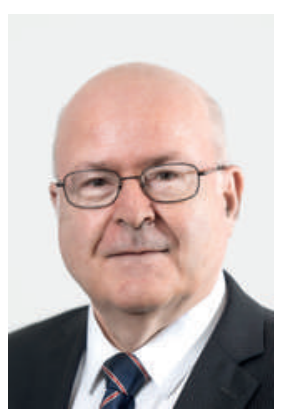

Auch während der heissen oder nassen Sommerwochen muss jede Ausgabe der Ärztezeitung ihr gutes Ende finden und zu einem guten Ende gehört natürlich ein «Zu guter Letzt». Zu einem «Zu guter Letzt» hinwiederum gehört ein Autor, der seine Ferienstimmung überwindet und einen Text verfasst, der möglichst ferienhaft leserfreundlich daherkommt.

Zunächst möchte ich einige lockere Überlegungen zu hidden agendas anstellen, weil mir solche immer wieder begegnen und weil sie sicher unzählige Verhandlungen unerkannt zum Teil entscheidend beeinflussen. Nicht so selten habe ich selber erlebt, dass man bei Sitzungen und bei der Erörterung von Projekten das Gefühl bekam, dass da über eine Struktur, über eine Zusammenarbeit, über ein Regelwerk, über Personelles diskutiert wurde, dass aber die eigentlichen Ziele, die eigentlichen Argumente und die eigentlichen Beweggründe gar nicht auf den Tisch kamen. Auch bei der Berichterstattung über politische Verhandlungen und Versuche der Konfliktlösung auf internationaler Ebene kommt immer wieder die Vermutung auf, dass die effektiven Interessen und die wahren Beweggründe zumindest während der offiziellen Gesprächsrunden nicht offengelegt werden. Vielleicht im Hinterzimmer? Dafür hagelt es dann Pressemitteilungen und Interviewantworten, mit welchen ein hocherfreuliches Ergebnis suggeriert wird, von dem die Geschichte uns dereinst erzählen wird, wie unerheblich es eigentlich war. Zur Verhandlungskunst gehört das Sensorium, solche hidden agendas zu erspüren, und ich wage zu behaupten, dass es durchaus auch Verhandlungen gibt, bei denen beide Parteien letztlich besser fahren würden, wenn die Agenden offengelegt würden. Auch in der Standes- und Gesundheitspolitik sind dafür Beispiele zu finden. Auf deren Erwähnung verzichte ich, möchte ich doch nicht Gefahr laufen, die heitere Ferienstimmung der Leserschaft einzutrüben.

Auch im Rahmen der Arzt-Patienten-Beziehung kann eine hidden agenda eine wichtige Rolle spielen. Lukas Zimmerli und seine Co-Autoren haben sie in einem Artikel [1] als «nicht deklarierte Beweggründe für einen Arztbesuch» definiert. "Dazu gehören Erwartungen, Gefühle, Ängste der Patienten, welche dem Arzt nicht ohne weiteres preisgegeben werden.» Deren Erkennen könnte fruchtlose Abklärungen verhindern, die mit dem Konsultationsgrund eigentlich nicht im Zusammenhang stehen.

Und nun zum verborgenen Phänomen, das in der Bildung eine Rolle spielen kann, dem hidden curriculum. Der Begriff wird seit den sechziger Jahren des vergangenen Jahrhunderts in der Pädagogik verwendet und bedeutet die meist ungewollte Vermittlung von Bildungselementen, die nicht in den Lehrplänen verzeichnet sind. Sie geschieht in erster Linie auf der Basis einer Diskrepanz zwischen gelehrtem und gelebtem Verhalten der Lehrenden. Es geht - und nun schauen wir auf die ärztliche Aus- und Weiterbildung - weniger um Kenntnisse und Fertigkeiten, sondern um die Persönlichkeitsbildung, um die Kommunikationsweise, um die Sozialisierung im Beruf, um das, was im angelsächsischen Raum mit professionalism bezeichnet wird.

Im Buch Understanding Medical Professionalism [2] wird das hidden cirriculum wie folgt beschrieben: «Lessons that are learned, but are not explicitly intended. These lessons may be contrary to the formal curriculum. The hidden curriculum is embedded in the organizational structure and culture and influences the norms and values that students and interns learn.» Auf der Basis einer Umfrage werden folgende Beispiele erwähnt: «Learn how to act like you know everything, whether or not you do.»

"It's about surviving, not excelling.»

«Politics matter - spend the most time with the most powerful person.»

«The attending [verantwortlicher Arzt] is right even when the attending is wrong.»

«Every attending will have different expectations about how to write a note and orders.»

Dieses hidden curriculum ist ein Phänomen, auf das wir sensibilisiert sein müssen und dem wir unbedingt auch Aufmerksamkeit schenken müssen, wenn die Sommerferienzeit vorbei ist. Klare Lernziele in den Weiterbildungsprogrammen und Logbüchern sind zwingend notwendig, doch gilt es auch, sich der Mechanismen bewusst zu sein, die der gesamten Entwicklung einer überzeugenden Arztpersönlichkeit im Wege stehen können. Nur wenn Lehren und Handeln kongruent sind, hat das hidden curriculum keine Chance. 